\title{
Turbulent Burning Velocity Measurements: Extended to Extreme Levels of Turbulence
}

\section{Timothy M. Wabel ${ }^{\star 1}$, Aaron W. Skiba ${ }^{1}$, James F. Driscoll ${ }^{2}$}

"Corresponding author

${ }^{1}$ Graduate Student Research Assistant, University of Michigan, Ann Arbor, MI 48109 USA

${ }^{2}$ Arthur B. Modine Professor, University of Michigan, Ann Arbor, MI 48109 USA

Abstract

Previous measurements of turbulent burning velocity $\left(S_{T}\right)$ have been reported by Gülder and colleagues for intense levels of turbulence, defined to be $u^{\prime} / S_{L}$ values between 12 and 24, and normalized integral scales $\left(L_{x} / \delta_{L}\right)$ up to 46 . The present work extends burning velocity measurements to much higher levels of turbulence than have been considered before: to extreme turbulence defined as $u^{\prime} / S_{L}$ values from 25 to 163 and $L_{x} / \delta_{L}$ up to 114 . These conditions are argued to be more representative of the turbulence found in certain engines. To do so, a new large, piloted Bunsen burner (called Hi-Pilot) was developed and $\mathrm{OH}$ and formaldehyde PLIF images provided the time-averaged contours of progress variable based on $\mathrm{OH}$ $\left(c_{O H}\right)$. The conventional global consumption speed $\left(S_{T, G C, 1} / S_{L}\right)$ is based on the $c_{O H}=0.5$ contour and it was found to exceed 25. Two other measured speeds are based on the leading edge $\left(S_{T, G, 2}\right)$ and the component due to flamelet surface density $\left(S_{T, F}\right)$. Varying the integral scale had a significant effect on $S_{T, G C, 2}$ but not on the other two burning velocities. The consumption speed $S_{T, G C, 1}$ curve displayed "bending" in the range of extreme turbulence, while the flamelet surface density contribution $\left(S_{T, F}\right)$ curve instead flattened out and was independent of turbulence intensity. A possible explanation for these measured trends is based on the observed extensive broadening of the preheat zone. Preheat broadening depends on the integral scale and is believed to attenuate the turbulence that eventually interacts with the reaction zone. Preheat broadening was also found to cause a breakdown of the thin flamelet assumption; this appears to cause thermal diffusivity to dominate over the flame wrinkling mechanism.

Turbulent Combustion Isep Burning Velocity Isep OH-PLIF IPACS 71.35.-y Isep 71.35.Lk Isep 71.36.+c \MSC[2008]23-557 


\section{Introduction}

The turbulent burning velocity $\left(S_{T}\right)$ is one metric that is commonly used to compare different experiments and simulations of premixed turbulent flames [1]. However, disagreements have arisen because it has several different definitions. Furthermore, although values of $S_{T}$ can be correlated if the experiments have the same general flame geometry [1] (such as a Bunsen geometry), attempts to correlate data from Bunsen, spherical and counter-flow flames have not been successful.

A research question is: how does $S_{T}$ scale with turbulence level and integral scale when the values of these two parameters are increased by a factor of six above the largest values previously achieved? Information about the flame structure and burning velocity within extreme turbulence is needed to improve numerical simulations, including those of Sankaran et al. [2], Sundaram et al. [3], Huh et al. [4], and Lee and Huh [5]. Kariuki [6] studied burning velocity for complex geometries, while Gülder has performed extensive work on Bunsen geometries.

Gülder and colleagues $[7,8,9,10]$ measured $S_{T}$ for the largest values of $u^{\prime} / S_{L}$ that had been achieved for Bunsen-type flames prior to the present study; their values ranged between 12 and 24, which is defined as intense turbulence. Their integral scale $\left(L_{x}\right)$ ranged from 0.5 to 3 $\mathrm{mm}$, and when normalized by flame thickness was as large as 46 . The present work extends the previous database by measuring turbulent burning velocity, as well as brush thickness and flame surface density, in the new range of $u^{\prime} / S_{L}$ from 25 to 163 , which is defined here to be extreme turbulence. Longitudinal integral scale was varied from 6 to $41 \mathrm{~mm}$, and when normalized by the preheat zone thickness $\left(L_{x} / \delta_{P H Z, L}\right)$ values as large as 114 were achieved. This extreme turbulence is argued to be more representative of certain engine conditions. Gülder and co-workers [7, 8] and Bradley, Mansour and co-workers [11, 12, 13, 14, 15] observed that large turbulence levels caused their burning velocity curves to display a 'bending' effect. They also observed that increasing the integral scale increased $S_{T}$. Both studies found that an improved correlation occurs if $S_{T} / S_{L}$ is plotted against the turbulent Reynolds number $\left(\operatorname{Re}_{T}=u^{\prime} L_{x} / v\right)$. The thickness of the preheat zone is another relevant parameter since it indicates the degree of turbulent diffusion upstream of the reaction layer. The preheat thicknesses of the present flames are reported in a separate paper [16] and are used here to help explain some of the observations. Correlations between turbulent flame speed and the aforementioned variables are an objective of this work.

\section{Experiment Details}


The Hi-Pilot is a highly turbulent, premixed, axisymmetric burner that stabilizes a $21.6 \mathrm{~mm}$ diameter central Bunsen flame. Premixed reactants are composed of methane-air at an equivalence ratio $\varphi=0.75$. The central flame is surrounded by a $108 \mathrm{~mm}$ co-flow of hot products produced by the same methane-air mixture. Further burner details are found in Ref. [16].

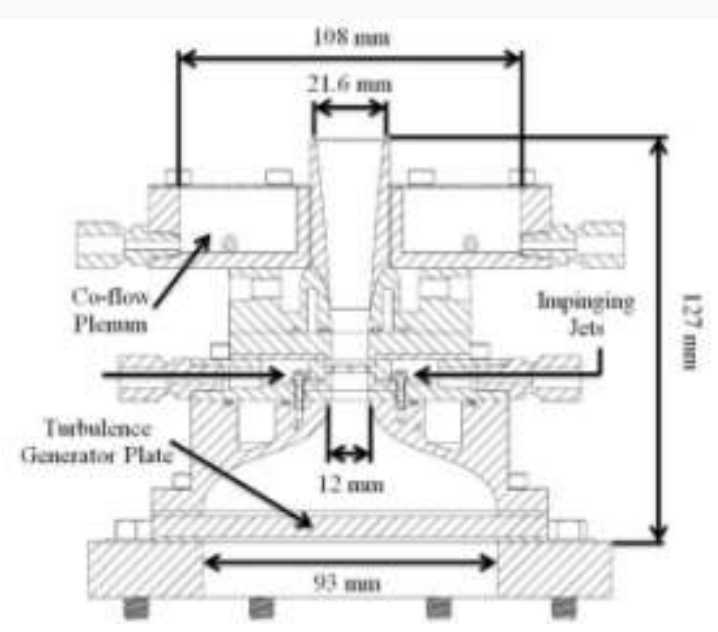

Figure 1 Schematic of the Michigan Hi-Pilot Burner.

Run conditions are listed in Table $1 \downarrow$; they were obtained by using slotted plates, a converging plenum [16], and a mean centerline velocity as large as $89 \mathrm{~m} / \mathrm{s}$. To normalize the data, values of laminar flame speed $\left(S_{L}\right)$ and preheat thickness $\left(\delta_{P H Z, L}\right)$ were determined from CHEMKIN to be $23.2 \mathrm{~cm} / \mathrm{s}$ and $0.36 \mathrm{~mm}$, respectively.

Table 1 Run conditions and parameters for the Hi-Pilot Experiment Case

$$
U_{0}(\mathrm{~m} / \mathrm{s}) \quad u^{\prime}(\mathrm{m} / \mathrm{s}) \quad L_{x}(\mathrm{~mm}) \quad R e_{T} \quad D a_{T} \quad u^{\prime} / S_{L} \quad L_{x} / \delta_{P H Z, L}
$$

\begin{tabular}{|c|c|c|c|c|c|c|c|}
\hline $1 a$ & 7.7 & 1.7 & 10 & 1,050 & 20 & 7.21 & 27 \\
\hline $2 a$ & 14 & 2.9 & 7.5 & 1,440 & 9.0 & 12.6 & 21 \\
\hline $3 a$ & 32 & 6.0 & 20 & 7,900 & 12 & 25.9 & 56 \\
\hline $4 a$ & 44 & 10 & 25 & 16,500 & 8.8 & 43.4 & 70 \\
\hline $5 a$ & 64 & 24 & 37 & 58,200 & 5.4 & 104 & 103 \\
\hline $6 a$ & 78 & 37 & 41 & 99,000 & 3.9 & 160 & 114 \\
\hline $1 b$ & 6.0 & 1.6 & 7.3 & 761 & 16 & 6.86 & 20 \\
\hline
\end{tabular}




\begin{tabular}{|c|c|c|c|c|c|c|c|}
\hline 2b & 9.2 & 3.1 & 7.8 & 1,580 & 8.8 & 13.4 & 22 \\
\hline 3b & 21 & 7.1 & 9.2 & 4,240 & 4.6 & 30.5 & 25 \\
\hline 4b & 28 & 9.3 & 8.4 & 5,050 & 3.2 & 39.8 & 23 \\
\hline $5 b$ & 45 & 20 & 10 & 13,300 & 1.9 & 84.2 & 29 \\
\hline $6 b$ & 72 & 29 & 12 & 22,300 & 1.4 & 126 & 32 \\
\hline cc & 8.5 & 1.2 & 6.1 & 475 & 18 & 5.16 & 17 \\
\hline 2c & 16 & 2.3 & 7.6 & 1,140 & 12 & 9.83 & 21 \\
\hline 3c & 32 & 5.7 & 9.3 & 3,420 & 5.8 & 24.4 & 26 \\
\hline $4 c$ & 44 & 9.0 & 10 & 5,980 & 4.0 & 38.6 & 28 \\
\hline $5 c$ & 64 & 25 & 19 & 31,700 & 2.6 & 109 & 53 \\
\hline $6 c$ & 89 & 38 & 17 & 40,900 & 1.5 & 163 & 46 \\
\hline
\end{tabular}

\subsection{Characterization of Turbulence and Flame Boundary}

Hotwire anemometer measurements showed that u' varied by less than $15 \%$ across the central $80 \%$ of the burner exit with no flame present. Laser Doppler Velocimetry (LDV) was used [16] to measure centerline values of mean velocity, $u$ ', and integral scale, as listed in Table $1 \uparrow$. Taylor's frozen flow hypothesis (which assumes a linear transformation between the temporal and spatial autocorrelations) has often been applied in the literature to determine integral length scales from single point measurements, even at very large turbulence levels. However, as the turbulence intensity becomes large Taylor's approximation becomes invalid. Since $u$ '/ $U_{0}$ exceeds $30 \%$ for many conditions studied here, a correction [17, 18, 19, 20, 21, 22, 23] was applied that relates the length scale based on Taylor's frozen flow hypothesis $L_{x, F R}$ to the true integral scale $L_{x}$ :

$$
\text { (1) } L_{x}=L_{x, F R}\left(1+5\left(u^{\prime} / U_{0}\right)^{2}\right)^{1 / 2}
$$

For a typical $u^{\prime} / U_{0}$ of $35 \%$, the correction causes a $27 \%$ increase in integral scale. This correction was applied, however none of the conclusions of the work are changed if it were not. 
A comment is required regarding the size of our longitudinal integral length scales, which in some cases approach twice the burner exit diameter. It is well-established that the longitudinal integral scale can exceed ten times the lateral integral scale when a high contraction ratio nozzle is used. Tennekes and Lumley [24] examined the conservation equations and showed that vortex stretching occurs when a strong axial velocity gradient exerts a strain rate that elongates eddies in the flow direction. The vortex stretching mechanism has been explained by Tsugé [25], Reynolds and Tucker [26] and Sreenivasan and Narasimha [27]. Both Ayyalasomayajula and Warhaft [28] and Grady et al. [29] measured longitudinal integral scales that increased by a factor of ten as the wall contraction ratio increased. In both studies the integral scales increased from 5-10 mm to $85-120 \mathrm{~mm}$. Menon has used LES [30] to simulate vortex stretching in a nozzle having an area contraction ratio of 9.3:1. He showed that many large eddies have a length approximately five times greater than their width. The present experiment has an area contraction ratio of 60:1.

The time-averaged flame boundary was determined from 400 simultaneous hydroxyl $(\mathrm{OH})$ and formaldehyde $\left(\mathrm{CH}_{2} \mathrm{O}\right)$ PLIF images. $\mathrm{OH}$ was excited with a $\mathrm{Nd}$ :YAG laser emitting $300 \mathrm{~mJ}$ at $532 \mathrm{~nm}$, which pumped a dye laser operating on Rhodamine 6G dye. The dye output at $568.14 \mathrm{~nm}$ was doubled to generate $\sim 4 \mathrm{~mJ}$ at $284.07 \mathrm{~nm}$. The sheet was $220 \mathrm{~mm}$ tall and $0.200 \mathrm{~mm}$ thick. A square field of view (FOV) between 150 and $200 \mathrm{~mm}$ per side (depending on the height of the flame) was imaged using an intensified CCD camera with a gate time of 100 ns and resolution of 250-350 $\mu \mathrm{m} /$ pixel. Background light was rejected using a bandpass filter centered at $310 \mathrm{~nm}$. Formaldehyde $\left(\mathrm{CH}_{2} \mathrm{O}\right)$ was excited by the $355 \mathrm{~nm}$ output of a second $\mathrm{Nd}$ :YAG laser. The $\mathrm{CH}_{2} \mathrm{O}$ fluorescence was collected with a second intensified CCD camera admitting light between 364 and $533 \mathrm{~nm}$, with similar FOV and resolution.

The resulting PLIF images were binarized using a local thresholding algorithm. This method was developed because of complexities arising from applying standard edge detection techniques in the presence of multiple pockets of reactants. Each pixel in the PLIF images was compared to a local maxima in signal intensity; pixels with an intensity less than $25 \%$ of the local maxima were set to zero. Although the selection of $25 \%$ was arbitrary, results do not change with different thresholds due to the sharp gradient in $\mathrm{OH}$ concentration near the flame front. This algorithm was iterated four times until convergence to the final binarized image was achieved. Canny edge detection was used to extract flame surface locations from the binary image. Figure $2 \downarrow$ presents an instantaneous flame surface (as well as the contour of average flame position, defined in section 2.3) and demonstrates the successful identification of reactant pockets, which is critical to accurate flame surface measurement. 


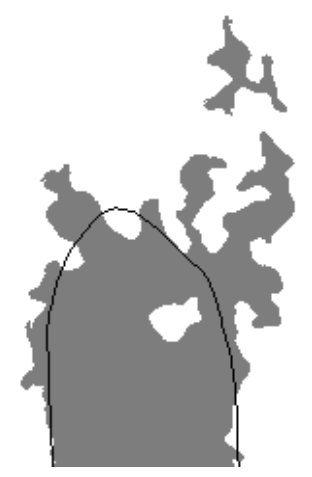

Figure 2 Example of an instantaneous flame surface for Case 3b (reactants in gray, products in white). The contour of average flame position is shown in black. This line indicates

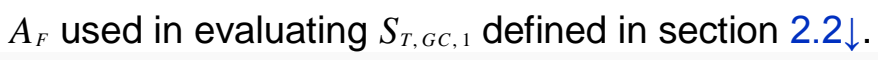

\subsection{Determination of Turbulent Burning Velocities}

Envelope flames are ones for which all of the reactants pass through a flame front, as in a Bunsen burner. For such geometries, the conventional global consumption speed $\left(S_{T, G C, 1}\right)$ typically is measured using [31]:

$$
\text { (2) } S_{T, G C}=\frac{\dot{m}_{r}}{\rho_{r} \bar{A}_{f}}
$$

The numerator of Eq. $(2 \uparrow)$ is the mass flow rate of reactants and the denominator contains the density of the reactants. $S_{T, G C, 1}$ is nearly inversely proportional to the height of the Bunsen flame. The subscript 1 here denotes the conventional consumption speed that is based on the surface area $\left(A_{F}\right)$ of the $c_{O H}=0.5$ contour. $c_{O H}$ is a form of the mean progress variable that is determined by time-averaging the instantaneous binarized $\mathrm{OH}$ signal, as was done by Shepherd and Cheng [32, 33]. An example of the mean progress variable of $\mathrm{OH}$ and an instantaneous flame surface is given in Figure $2 \uparrow$ for a representative flame. The 3-D flame area was calculated by revolving the 2-D ${ }_{\text {ОН }}$ interface about the centerline and applying a standard formula for the area [34]. Another burning velocity $\left(S_{T, G C, 2}\right)$ was determined in the same way, except that the area of the $c_{O H}=0.2$ contour was used.

A third burning velocity was defined by Bray and Cant [35] to be:

$$
\text { (3) } \frac{S_{T, F}}{S_{L}}=\frac{1}{L} I_{0} \int_{-\infty}^{\infty} \int_{-\infty}^{\infty} \Sigma d \eta d \xi
$$


$\Sigma$ is the flame surface density that is integrated over $\eta$ (the flame-normal) and $\xi$ (the flame-tangent direction). $I_{0}$ is the stretch factor [1] that is nearly unity in this work and $L$ is flame length. The velocity $S_{T, F}$ is argued to be only one component of the entire burning velocity, because the derivation of Eq. (3个) assumes that only laminar flamelets exist, and that they propagate locally at the laminar flame speed. To measure $\Sigma$ a $1 \mathrm{~mm}^{2}$ interrogation box was selected. The time-averaged perimeter of the $\mathrm{OH}$ boundary in the box was divided by the area of the box.

\section{Results}

\subsection{Global Consumption Speeds}

Measurements are plotted in Figure $3 \downarrow$ a of the conventional global consumption speed, $S_{T, G C, 1}$ that is based on the $c_{O H}=0.5$ contour. The maximum value of $u^{\prime} / S_{L}$ is 160 where $S_{T, G C, 1} / S_{L}$ reaches 27. Also included are data points from Yuen et al. [9], which had reported the largest previous burning velocities in Bunsen flames. It has been suggested that at large turbulence levels the burning velocity curve will flatten out and no longer increase with turbulence intensity. The current work shows that for the Bunsen flame this is not the case. Consumption speed is always increasing in Figure $3 \downarrow$, despite the bending that occurs as turbulence intensity $u$ ' $/ S_{L}$ exceeds 40 .
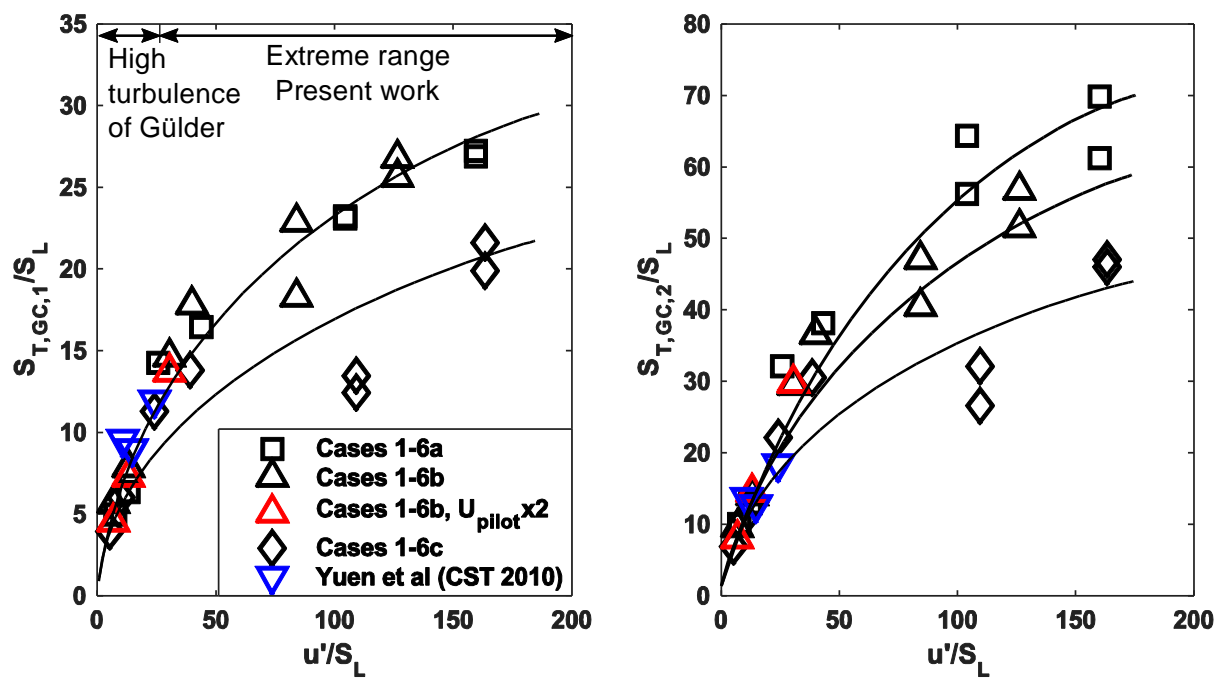

Figure 3 Global consumption speed at extreme levels of turbulence ( $u$ '/ $S_{L}$ between 25 and 160). Gülder's data for $u^{\prime} / S_{L}<25$ also shown. (a) standard consumption speed based on $c_{O H}=0.5$ contour, (b) consumption speed based on $c_{O H}=0.2$ contour. 
Figure $3 \uparrow b$ shows that if the burning velocity instead is defined using the $c_{O H}=0.2$ contour, the resulting values of $S_{T, G C, 2}$ are larger than $S_{T, G C, 1}$ in Fig. $3 \uparrow$ a. Also $S_{T, G C, 2}$ in Fig. $3 \uparrow$ b increases with increasing integral scales, while values of $S_{T, G C, 1}$ do not. It is observed that the differences due to integral scale are primarily observed at large turbulence intensity; the differences at $u^{\prime} / S_{L}<20$ are less significant. The cases with the smallest slotted turbulence-generating plate (condition 'c', indicated by diamonds) produce the smallest global consumption speeds. This is believed to be due to the different velocity profile this slot produces; Table $1 \uparrow$ shows that the centerline velocity $U_{0}$ for Case $6 \mathrm{c}$ is $14 \%$ larger than $6 \mathrm{a}$. This is seen to produce a much taller flame, and thus lowers the global consumption speed.

To explain the integral scale dependence in Fig. $3 \uparrow$, a first hypothesis is that larger eddies might be better able to displace the instantaneous flame surface from its mean location, and therefore produce a broader turbulent flame brush. This would lead to a smaller area of the leading edge and cause the global consumption speed to be larger for larger integral scales. However, it was found that the normalized centerline brush thickness $\left(\delta_{B} / D\right)$ collapsed to a linear function (see Figure $4 \downarrow$ ) of the normalized characteristic flame height $(H / D)$ indicating that the centerline brush thickness is not strongly dependent of integral scale. 


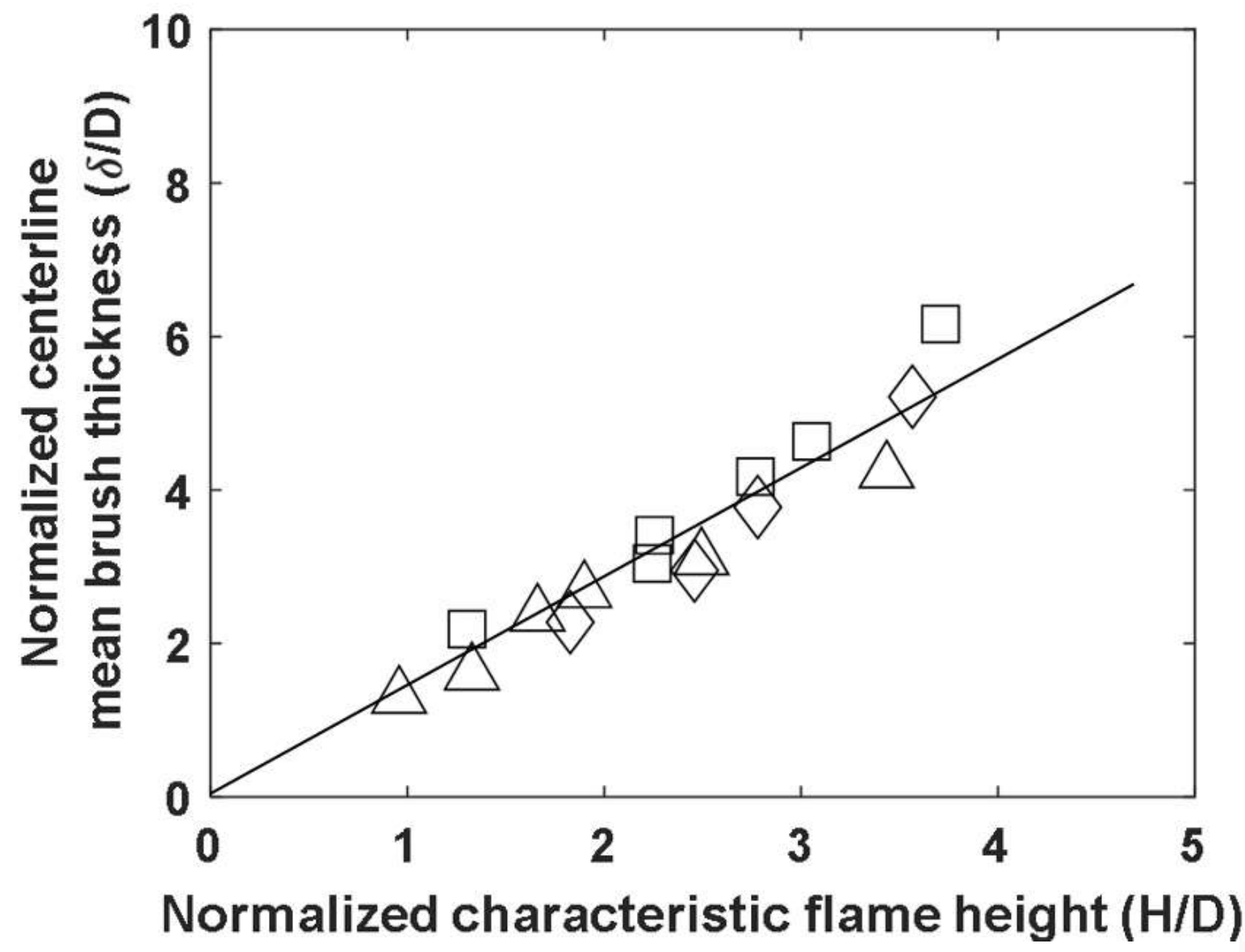

Figure 4 Normalized flame brush thickness versus normalized flame height $(H / D)$, where $D$ is the burner exit diameter. Symbols are the same as for Fig. $3 \uparrow$.

A more likely explanation of the integral scale dependence in Figure $3 \uparrow$ is that larger integral scales were observed to cause very large but relatively infrequent side-to-side displacements of the flame. This lateral displacement causes products to be located upstream of where the flame brush is normally positioned, thus reducing the effective flame area and increasing the measured burning velocity. Lateral motions are not found to alter the $c_{O H}=0.5$ contour. However the $c_{O H}=0.2$ contour is very near the exit plane at large radial distances from centerline, reducing the flame surface area used in $\mathrm{S}_{\mathrm{T}, \mathrm{GC}, 2}$ and explaining the differences between Figs. $3 \uparrow a$ and $3 \uparrow b$. An important conclusion is therefore that flame height alone does not give an accurate measure of burning velocity in these conditions, as a cone or parabola fitted to the measured flame heights would not capture these variations.

To evaluate the effect of the shear layer interaction with the central jet, Cases 1-3b were studied case with a co-flow velocity twice the standard conditions (accounting for thermal expansion the mass-average velocity was increased from 9 to $19 \mathrm{~m} / \mathrm{s}$ ). The results are given in 
Figure $3 \uparrow$, red open triangles indicating the doubled co-flow condition. The results show little change from the nominal condition, with Case 1 burning velocities reduced between $10-15 \%$ and Cases $2-3$ by less than $5 \%$, indicating the flame sits inside the shear layer and co-flow velocity does not strongly influence $S_{T, G C}$.

The bending of the curves in Figure $3 \uparrow$ is found to occur when the preheat zone thickness becomes very large (typically greater than $20 \mathrm{~mm}$, see Figure 5) [16]. The gray region in Fig. 5 is the preheat zone, which is identified by the presence of formaldehyde PLIF signal but no $\mathrm{OH}$ signal. CHEMKIN computations show that formaldehyde diffuses throughout the preheat zone in a laminar flame. The preheat zone in Fig. 5 begins where formaldehyde is $35 \%$ of its maximum value (corresponding to approximately $500 \mathrm{~K}$ ) and ends where the reaction zone (marked by $\mathrm{OH}$ signal) begins. Based on this observation, it is believed that one factor that affects the bending is the ratio of the integral scale to the preheat thickness. If the preheat thickness is 20 $\mathrm{mm}$ and the largest eddies are $6 \mathrm{~mm}$, it is unlikely that the smallest eddies will survive passage through the hot viscous preheat gases, and therefore cannot act on the flame surface. Furthermore, the response of the largest eddies in the flow is not known. Thus if the eddies do not survive, that may be a reason why increasing the turbulence level does not linearly increase the burning velocity and bending is observed. Moreover, cases with a larger integral scale see less 'bending' in Fig. $3 \uparrow b$, while smaller integral scales begin to exhibit a plateau in flame speed more rapidly, suggesting larger integral scales are more resistant to preheat-turbulence attenuation. Our observed result was predicted by Lipatnikov, who estimated that only eddies larger than the preheat zone thickness can survive the dissipative effects of the preheat region and modify the reaction layer and local burning rate [36]. 


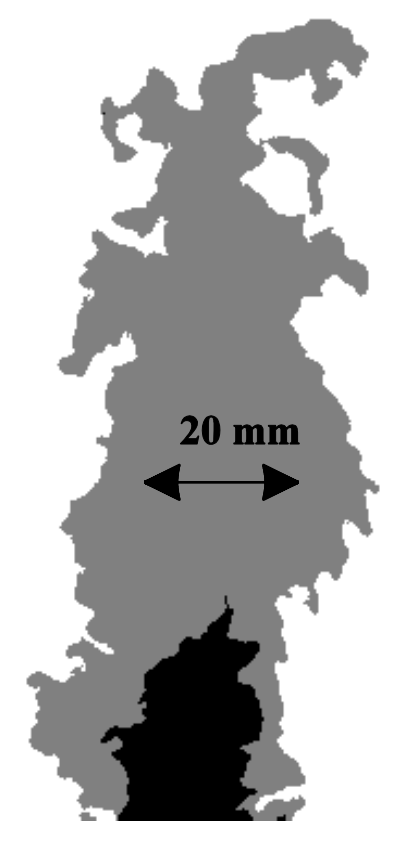

Figure 5 Illustration of broadening of the preheat zone for a representative flame (case 4a). Black indicates unburnt reactants, gray is preheated reactants (formaldehyde signal), and white is products.

This concept of eddy decay in the preheat zone can be stated in another way. Where the curves in Fig. 2 are linear, the preheat zone is thin so the turbulence level approaching the flame is nearly the same as the turbulence that traverses the thin preheat layer and reaches the reaction layer. Where the curves in Figure 3 are bending, the turbulence level ( $u$ ') that survives and reaches the reaction layer is less than the upstream value of $u$ ' that is plotted on the horizontal axis. The effective turbulence acting on the reaction layer has been reduced by the preheat layer. This would shift the data points for large u' to the left in Figure 3, resulting in less (or no) bending. The work suggests that a new type of turbulence should be considered, called the effective turbulence, that differs from the upstream turbulence which is measured ahead of the preheat region.

\subsection{Contribution Due to Flamelet Wrinkling}

The contribution due to flamelet wrinkling $\left(S_{T, F}\right)$ was defined in Eq. $(3 \uparrow)$ and is plotted as the lower curve in Figure $6 \downarrow$. It is seen to be much smaller than the consumption speed $S_{T, G C, 1}$ (which is reproduced from Figure $3 \uparrow$ as the upper curve in Figure $6 \downarrow$ ). The lower curve is flat, indicating that $S_{T, F}$ is independent of $u^{\prime} / S_{L}$. This trend was previously seen by Gülder et al. [8] and Yuen et al. [9] for lower turbulence levels. It can be explained by the transition from thin to 
broadened flamelets. Despite the flame continuing to consume reactants at a greater rate, the total wrinkled flame area remains unchanged. That is, the peak flame surface density decreases, but the width of the flame brush increases. Expressed another way, the flame occupies a larger region of space, but the probability of a flame existing at any given location decreases; this leads to an integrated flame-surface density that is constant. The contribution due to flamelet wrinkling $\left(S_{T, F}\right)$ is only useful as a burning velocity in the thin flamelet regime when it nearly equals the consumption speed. Since it contributes so little in Figure $6 \downarrow$ it is argued that $\left(S_{T, F}\right)$ is not useful for extreme turbulence. One explanation for the large difference between $S_{T, G C, 1}$ and $S_{T, F}$ is that at higher turbulence intensities the increase in turbulent diffusivity $\left(D_{T} \sim u^{\prime} L_{x}\right)$ is more important than flamelet wrinkling.

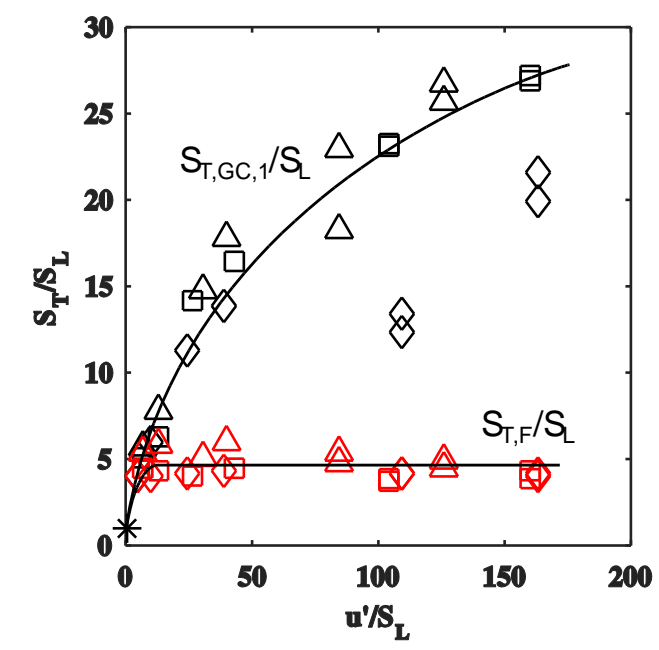

Figure 6 Mean flamelet velocity ( $S_{T, F}$, lower curve), compared to global consumption speed $\left(S_{T, G C, 1}\right.$, upper curve). Symbol notation is same as Fig $3 \uparrow$.

\subsection{Data Correlations}

A conventional idea is that flamelet wrinkling and the resulting increase in flame surface area is the primary contribution to burning velocities at lower turbulence intensities. Kuo examines a geometric relation for burning velocity originally derived by Shchelkin [37], who assumed the wrinkling process can be viewed as flame surfaces becoming distorted into cones. The result is of the form:

$$
\text { (4) } \frac{s_{T}}{s_{L}}=\sqrt{1+A\left(\frac{u^{\prime}}{s_{L}}\right)^{2}}
$$


In Eqn. (4个) $A$ is an experimentally determined constant. Bending (nonlinear behavior) has usually been observed in experiments [1, 9, 12,31], and is not accounted for in this analysis. Possible causes for this effect are flame surface destruction by merging or local extinction, residence time effects (associated with increasing the overall turbulence level $u$ ' by increasing the bulk velocity $U_{0}$ ), or turbulence attenuation through a broadened preheat zone. The geometric analysis of Shchelkin predicts a linear relationship between burning velocity and turbulence intensity at large values of $u^{\prime} / S_{L}$.

Figure 6 provides evidence that increases in burning velocity at large turbulence levels must be due to factors other than flamelet wrinkling. References [37, 40] discuss Damköhler's prediction that the turbulent burning velocity in the Broadened Preheat - Thin Reaction layers (BP-TR) regime is proportional to the square root of turbulent diffusivity $\left(u^{\prime} L_{x}\right)$ multiplied by a reaction rate, based on analogy with the laminar case:

$$
\text { (5) } \frac{S_{T}}{S_{L}}=\frac{\sqrt{\left(\alpha+u^{\prime} L_{x}\right) R R_{T}}}{\sqrt{\alpha R R_{L}}} \sim \sqrt{\frac{u^{\prime} L_{x}}{v}} \sqrt{\frac{R R_{T}}{R R_{L}}}
$$

Eq. (5个) predicts that integral scale is a governing parameter. However the ratio of reaction rates in Eq. (5个) can depend on flame stretch and the residence time during which the stretch rate is applied. This can introduce Karlovitz and Lewis numbers, as discussed by Andrews et al. [11], as well as the residence time $\left(x / U_{0}\right)$ of eddies in the flame brush that is based on the mean velocity. The following empirical relations are suggested, assuming that the reaction rate is unchanged by turbulence.

$$
\begin{gathered}
\text { (6) } \frac{S_{T, G C}}{S_{L}}=\sqrt{1+c_{1}\left(\frac{u^{\prime}}{S_{L}}\right)^{2}}\left(\frac{c_{2}}{c_{2}+\left(\frac{u^{\prime}}{s_{L}}\right)}\right)+c_{3} \sqrt{\left(\frac{u^{\prime}}{s_{L}}\right)\left(\frac{L_{x}}{\delta_{P H Z, L}}\right)\left(\frac{L_{y}}{\delta_{P H Z}, L}\right)}\left(\frac{\delta_{P H Z, T}}{\delta_{P H Z, L}}-1\right) \\
\text { (7) } \frac{\delta_{P H Z, T}}{\delta_{P H Z, L}}=1+c_{4}\left(\frac{u^{\prime}}{S_{L}}\right)^{n} *\left(\frac{L_{x}}{\delta_{P H Z, L}}\right)^{m} *\left(\frac{L_{y}}{\delta_{P H Z}, L}\right)^{p}
\end{gathered}
$$

Parameters $c_{1}-c_{4}, \mathrm{n}, \mathrm{m}$ and $\mathrm{p}$ for the present data are given in Table $2 \downarrow$. Note that values are listed that yield good curve fits for measured values of both consumption speeds $S_{T, G C, 1}$ and $S_{T, G C, 2 .}$. The square root factor that contains $c_{1}$ in Eqn. $6 \uparrow$ is identical to Eqn. $4 \uparrow$, and this factor dominates at low turbulence levels. The factor that contains $c_{2}$ is a bending term that is unity at low turbulence levels and becomes important at elevated turbulence levels. This factor is suggested by the analysis of Duclos et al. [41]; it arises due to the destruction term associated with flamelet merging in their flame surface density balance. The term that includes $c_{3}$ in Eqn. $6 \uparrow$ is seen to be negligible when the preheat zone thickness $\left(\delta_{P H Z, T}\right)$ is not much larger than its 
laminar value. For thick preheat zones, this term accounts for the enhanced diffusivity of heat due to turbulence within the preheat zone, as was suggested by Damköhler.

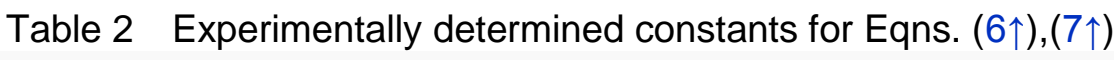

\begin{tabular}{c|c|c|c} 
Parameter & $S_{T, G C, 1} / S_{L}$ & $S_{T, G C, 2} / S_{L}$ & $\delta_{P H Z, T} / \delta_{P H Z, L}$ \\
\hline$c_{1}$ & 0.9 & 4 & - \\
\hline$c_{2}$ & 23 & 23 & - \\
\hline$c_{3}$ & $3 \times 10^{-4}$ & $3 \times 10^{-3}$ & - \\
\hline$c_{4}$ & - & - & 5 \\
\hline $\mathrm{n}$ & - & - & 0.30 \\
$\mathrm{~m}$ & - & - & 0.15 \\
$\mathrm{p}$ & & - & -0.11
\end{tabular}

It is expected that both the longitudinal and transverse integral scales are relevant. However, information regarding the transverse scales cannot be obtained from the LDV measurements. The analysis therefore uses the slot width of the turbulence generating plate as an estimate of the transverse length scale, $L_{y}$. In general, the quantities in Table $2 \uparrow$ will be functions that depend on the composition of the reactants. Since composition was not varied in this work, these parameters are constants.

The present results suggest that the turbulence level and integral scale at the reaction layer differ from those in the upstream reactants. This has been predicted by the very high $K a$ DNS flames of Savard et al. [42]. It is observed that our extreme levels of turbulence ( $u^{\prime} / S_{L}$ up to 163 ) broaden the preheat layer dramatically to a thickness that is regularly greater than 20 $\mathrm{mm}$. The elevated viscosity could reduce $u^{\prime}$ and modify $L_{x}$. This is a dynamic system, as the preheat thickness depends on the same variables as burning velocity; as the preheat layer broadens with increasing turbulence it attenuates the eddies acting on the reaction layer downstream. Thus it is suggested that the preheat zone thickness is a variable that indirectly modifies the burning velocity by attenuating the incoming turbulence. The work points out the need to measure the variation of turbulence level and integral scale as a function of distance through a thickened preheat zone, which has not been accomplished to date. 


\section{Conclusions}

1. Three types of turbulent burning velocity were measured in the new range of extreme turbulence (defined as $u^{\prime} / S_{L}$ between 25 and 163), which had not been studied before. The conventional consumption speed $S_{T, G C, 1}$ is based on the area of the $c_{O H}=0.5$ contour, while $S_{T, G C, 2}$ is based on the $c_{O H}=0.2$ contour and $S_{T, F}$ is the contribution of flamelet wrinkling that is determined by integrating the flame surface density. The curve of $S_{T, G C, 1} / S_{L}$ displayed bending and values exceeded 25 .

2. The curve of $S_{T, F}$ was flat, indicating that increased turbulence does not cause increased wrinkling or increased surface density. This is consistent with data of Gülder and others who considered lower turbulence levels. Thus $S_{T, F}$ is not a good metric in the extreme turbulence range because $S_{T, F}$ is based on thin flamelet assumptions that are no longer valid.

3. The role of integral scale was measured by varying $L_{x}$ over a wide range (6 $\mathrm{mm}$ to 41 $\mathrm{mm}$ ). Increased $L_{x}$ increases $S_{T, G C, 2}$ but not $S_{T, G C, 1}$ or $S_{T, F}$. This difference is explained by images of $\mathrm{OH}$ contours. Larger integral scales cause strong lateral motions that force lower regions of the flame to move upstream, and this affects $S_{T, G C, 2}$ more than $S_{T, G C, 1}$. Varying the integral scale has a larger effect in the extreme turbulence range than for smaller levels of turbulence.

4. The 'bending' effect is observed when the preheat layers become thick, suggesting that the thickened preheat layer plays a role in the bending. To explain several observations, it is hypothesized that increasing the turbulence into the extreme range $\left(u^{\prime} / S_{L}\right.$ from 25 to 163) causes the preheat zone to become so thick that eddies passing through the layer cannot survive. Therefore, burning velocity no longer increases linearly, resulting in the bending effect. The work suggests that the relevant turbulence considered should be the effective turbulence that is actually acting on the flame front.

5. OH and formaldehyde PLIF diagnostics offer improvements over Rayleigh or Mie scattering in that they confirm that the flame tip is closed and that any local extinction is insignificant. Correct measurements of consumption speed depend on this confirmation.

\section{Acknowledgments}

Support for this research was provided by AFOSR Grant FA9550-12-1- 0101; additional support was provided by National Science Foundation grant CBET 0852910. 


\section{References}

\section{References}

[1] J. F. Driscoll, Prog. Energy Comb. Science 34 (2008) 91-134

[2] R. Sankaran, E.R.Hawkes, C.S. Yoo, J.H. Chen, Combust. Flame 162, 9 (2015) 3294-3306

[3] B. Sundaram, A.Y. Klimenko, M.J. Cleary, U. Maas, Proc. Combust. Inst. 35 (2015) 15171525.

[4] K. Huh, J. Kwon, D. Lee, Physics of Fluids 25, 7 (2013) 075108.

[5] D. Lee, K. Y. Huh, Combust. Flame 159, 4 (2012) 1576-1591.

[6] J. Kariuki, JR Dawson, E Mastorakos, Combust. Flame 159, 8 (2012) 2589-2607.

[7] Ö. L. Gülder, Proc. Comb. Inst. 23 (1990) 743-750

[8] Ö. L. Gülder, , G. J. Smallwood, Comb. Sci. Tech. 179 (2007) 191-206

[9] F. Yuen, Ö. L. Gülder, Comb. Sci. Tech. 182 (2010) 544-558

[10] P. Tamadonfar, Ö. L. Gülder, Comb. Flame 161 (2014) 3154-3165

[11] G. Andrews, D. Bradley, S. Lwakabamba, Comb. Flame 24 (1975) 285-304

[12] D. Bradley, A. Lau, M. Lawes, Phil. Trans: Phys. Sci. Engin. 338 (1992) 359-387

[13] R. Abdel-Gayed, D. Bradley, Phil. Trans. Roy. Soc. Lond. A. Vol. 301 No. 1457 (1981) 1-25

[14] D. Bradley, M. Lawes, K.X. Liu, M.S. Mansour, Proc. Combust. Inst. 34, 1, (2013) 15191526.

[15] D. Bradley, M. Lawes, M.S. Mansour, Flow Turb. Combust. 87, 2-3 (2011) 191-204. 
[16] J. E. Temme, T. M. Wabel, A. W. Skiba, J. F. Driscoll, 53rd AIAA Aerospace Sciences Meeting, AIAA Paper 2015-0168 (2015). An updated and modified version is submitted to the 36th Symposium on Combustion, 2015.

[17] M. Fisher, P. Davies, J. Fluid Mech. 18 (1964) 97-116

[18] G. Heskestad, J. Applied Mech. 32, 4 (1965) 735-739

[19] H. Wu, G. Patterson, Chem. Engin. Sci. 44, 10 (1989), 2207-2221

[20] S. Kresta, P. Wood, Chem. Engin. Sci. 48, 10 (1993), 1761-1774

[21] E. Wernersson, C. Tragardh, Exp. Fluids 28 (2000), 532-545

[22] C. Garcia, M. Garcia, Exp. Fluids 41 (2006) 91-101

[23] N. R. Panchapakesan, J. L. Lumley, J. Fluid Mech. 246 (1993) 197-223

[24] Tennekes H, Lumley J (1972) A first course in turbulence, page 83. The MIT press, Cambridge.

[25] S. Tsugé, "Effects of flow contraction on evolution of turbulence," Phys. Fluids 27, 1948, 1984.

[26] J. Reynolds and H. J. Tucker, "The distortion of turbulence by general uniform irrotational strain,” J.Fluid Mech. 68, 6731975.

[27] K. R. Sreenivasan and R. Narasimha, "Rapid distortion of axisymmetric turbulence," J. Fluid Mech. 84, 4971978 .

[28] S. Ayyalasomayajula and Z. Warhaft, "Nonlinear interactions in strained axisymmetric highReynolds-number turbulence," J. Fluid Mech. 566, 2732006.

[29] N. Grady, R.W. Pitz, B. Ochs, T. Slais, D. Scarborough, S. Menon, Propagation of Premixed Flame Kernels in High Speed Channel Flows with Moderate Turbulence, AIAA Paper 2015-0169.

[30] S. Menon, LES, private communication (used with permission). See supplementary material. 
[31] S. A. Filatyev, J. F. Driscoll, C. D. Carter, J. M. Donbar, Combust. Flame 141 (2005) 1-21

[32] I. Shepherd, R. Cheng, Combust. Flame 127 (3) (2001) 2066-2075

[33] R. Cheng, I. Shepherd, B. Bedat, L. Talbot, Comb. Sci. Tech. 174 (2002) 29-59

[34] K. Singh, Engineering Mathematics (6 ed). Tata McGraw-Hill. Chapter 6 page 90.

[35] K. N. C. Bray, R. S. Cant, Proc. R. Soc. Lond. A 434 (1991) 217-240

[36] A.N. Lipatnikov, J. Chomiak, Prog. Energy Comb. Science 28 (2002) 1-74

[37] K. K. Kuo, R. Acharya, Fundamentals of Turbulent and Multiphase Combustion (2012), John Wiley \& Sons, Inc.

[38] F. A. Williams, Prog. Energy Comb. Science 26 (2000) 657-682

[39] A. J. Aspden, M. S. Day, J. B. Bell, J. Fluid Mech. 680 (2011) 287-320

[40] N. Peters, Turbulent Combustion. Cambridge University Press, Cambridge UK, 2000.

[41] J. M. Duclos, D. Veynante, T. Poinsot, Combust. Flame 95 (1993) 101-117

[42] B. Savard, B. Bobbitt, G. Blanquart, Proc. Combust. Inst. 35 (2015) 1377-1384 